\title{
artigo
}

Santos, A.L.T.; Souza, J.F.; Ribeiro, C.L.; Nascimento, P.A.C.; Bruno, T.; Cecília, H.

Prevalência de transtornos mentais menores em estudantes da área da saúde e os fatores relacionados

\section{Prevalência de transtornos mentais menores em estudantes da área da saúde e os fatores relacionados}

\author{
Prevalence of minor mental disorders in health students and related factors \\ Prevalencia de trastornos mentales menores en estudiantes de salud y factores afines
}

\begin{abstract}
RESUMO
Objetivo: Esse estudo foi investigar prevalência de transtornos mentais menores em estudantes da área da saúde, e os fatores de risco que facilitam para o desencadeamento dos mesmos. Método: 0 presente estudo é do tipo Revisão Integrativa da Literatura, foi realizada no período de fevereiro a dezembro de 2020, os artigos selecionados para fundamentação do estudo em questão foram buscados nas seguintes bases de dados LILACS e ScieLO. Resultados: Foram identificadas duas categorias I- Prevalência dos transtornos mentais comuns em universitários da área da saúde. II- Fatores associados ao risco de depressão e ansiedade no ambiente universitário. Conclusão: E com esse estudo foi possível evidenciar a prevalência dos transtornos mentais comuns como depressão e ansiedade entre os estudantes universitários, e os fatores de risco associados a esse evento tais como pressão acadêmica e sobrecargas, insatisfação com o curso, relacionamentos insatisfatórios com os colegas e docentes e residir longe da família.
\end{abstract}

DESCRITORES: Saúde mental; Universitário; Distúrbios emocionais; Fatores de risco; Transtorno mental.

\section{ABSTRACT}

Objective: this study was to investigate the prevalence of minor mental disorders in health students, and the risk factors that facilitate their triggering. Method: the present study is of the Integrative Literature Review type, it was carried out from February to December 2020, the articles selected to support the study in question were searched in the following LILACS e ScieLO. Results: Two categories were identified I- Prevalence of common mental disorders in university students in the health field. II- Factors associated with the risk of depression and anxiety in the university environment. Conclusion: And with this study it was possible to evidence the prevalence of common mental disorders among university students, and the risk factors associated with this event such as academic pressure and overload, dissatisfaction with the course, unsatisfactory relationships with colleagues and teachers and residing away from the family.

DESCRIPTORS: Mental health; University; Emotional disorders; Risk factors; Mental disorder.

\section{RESUMEN}

Objetivo: este estudio fue investigar la prevalencia de trastornos mentales menores en estudiantes de salud y los factores de riesgo que facilitan su desencadenamiento. Método: el presente estudio es del tipo Revisión Integrativa de Literatura, se realizó de febrero a diciembre de 2020, los artículos seleccionados para sustentar el estudio en cuestión fueron buscados en las siguientes bases de datos LILACS e ScieLO. Resultados: Se identificaron dos categorías I- Prevalencia de trastornos mentales comunes en estudiantes universitarios del campo de la salud. II- Factores asociados al riesgo de depresión y ansiedad en el ámbito universitario. Conclusión: Y con este estudio se pudo evidenciar la prevalencia de trastornos mentales comunes entre estudiantes universitarios, y los factores de riesgo asociados a este evento como presión y sobrecarga académica, insatisfacción con el curso, relaciones insatisfactorias con colegas y docentes y residente de la familia.

DESCRIPTORES: Salud mental; Universidad; Trastornos emocionales; Factores de riesgo; Trastorno mental.

RECEBIDO EM: 26/02/2021 APROVADO EM: 08/03/2021

\section{Ana Letícia Torres dos Santos}

Graduanda do curso de enfermagem pela Faculdade de Medicina de Juazeiro do Norte(FMJ).

ORCID: 0000-0001-7111-2905 


\section{Joíce Fabricio de Souza}

Mestranda em Saúde Coletiva na Universidade de Fortaleza. - PPGSC/UNIFOR. Bolsista pela Fundação Cearense de Apoio ao Desenvolvimento Científico e Tecnológico- FUNCAP.

ORCID: 0000-0002-3165-1135

\section{Camila Lima Ribeiro}

Mestranda em Saúde Coletiva- PPGSC/UNIFOR- Universidade de Fortaleza. Especialista em Ginecologia e Obstetrícia - UNIQ. Especializanda em Enfermagem Forense -Unyleya.

ORCID: 0000-0002-1599-8454

\section{Polyana Amorim Cruz Nascimento}

Docente do curso de Medicina e enfermagem na Faculdade de Medicina Estácio de Juazeiro do Norte. Mestre em gestão em saúde pela universidade estadual do Ceará. Especialista em gestão da clínica e educação na saúde (Pelo sírio Libanês).

ORCID: 0000-0002-9899-0932

\section{Thiago Bruno}

Docente na Faculdade de Medicina Estácio de Juazeiro do Norte. Especialista em UTI, Urgência e Emergência e em Saúde da Família. ORCID: 0000-0003-1904-9085

\section{Halana Cecília}

Docente na Faculdade de Medicina Estácio de Juazeiro do Norte. Especialista em Saúde Pública, Mestre em Educação. ORCID: 0000-0001-8049-1994

\section{INTRODUÇÃO}

D evido á almejada formação profissional, a vida universitária geralmente é um momento de satisfação, porém muitos não estão completamente preparados pra enfrentar esse desafio, encontrando muitas vezes dificuldade de se adaptar a vida acadêmica devido a grandes mudanças e acabam sofrendo com isso $^{1}$. Estudos mostram que universitários principalmente da área da saúde sofrem com transtornos mentais comuns (TMC) como a depressão e a ansiedade ${ }^{2}$.

Segundo a Organização Mundial da Saúde (OMS), o suicídio gera $800 \mathrm{mil}$ mortes por ano no mundo, sendo a segunda maior causa de morte entre jovens de 15 a 29 anos, justamente a faixa etária de estudantes prestes a ingressar na faculdade ou que já estão cursando³. Estudos recentes de base nacional e escolar mostraram que $30 \%$ dos adolescentes apresentavam transtornos mentais comuns, identificados por sintomas de depressão, ansiedade e queixas somáticas inespecíficas ${ }^{4}$.

A ansiedade e a depressão são os transtornos mais comuns na vida dos universitários. A depressão é uma doença caracterizada por alteraçôes do humor, insônia, perda de energia e fadiga, falta de auto cuidado, desinteresse em geral, enquanto a ansiedade que também é um transtorno mental caracteriza-se por distúrbios de sono, fadiga e tremores. Ambos podem ser desencadeados por pressão emocional, medo, insegurança, estresses e grandes responsabilidades, o que é recorrente no ambiente acadêmico ${ }^{5}$.

Diagnosticar para tratar os transtornos mentais o quanto antes é imprescindível para o bem-estar mental na vida dos universitários. Grande parte dos estudos sugere uma abordagem terapêutica que contemple tanto medicamentos como psicoterapia visando uma qualidade de vida positiva. Além destes citados anteriormente é considerável destacar a importância da prevenção através de debates, palestras, ou seja, divulgação de informação ${ }^{6}$.

Tendo em vista o que foi exposto no presente estudo, cabe destacar o seguinte questionamento: Até onde a vida acadêmica pode prejudicar o psicológico dos universitários a ponto de ocasionar distúrbios mentais e emocionais?

Problemas mentais podem gerar prejuízos à vida das pessoas acometidas. Portanto é necessário abranger ainda mais assuntos envolvendo a saúde mental no ambiente acadêmico através de palestras e debates focando na identificação, preven- ção e tratamentos dos transtornos mais comuns. É importante investigar a percepção dos universitários sobre a vida acadêmica e suas associações diante do sofrimento psíquico. Considera-se que para identificar fatores de proteção com o objetivo de favorecer o bem estar dos alunos e tornar suas vivencias mais positivas no ambiente acadêmico, é necessário explanar a importância do planejamento de intervenções e fazer a inclusão de aspectos psicológicos como coping e resiliência, assim a faculdade tende a ter uma abordagem mais integral, não apenas acadêmica e profissional ${ }^{2}$.

Assim o objetivo principal desse estudo foi investigar através da literatura a prevalência dos transtornos mentais menores em estudantes da área da saúde.

\section{MÉTODO}

Trata-se de uma revisão integrativa, do tipo exploratório descritiva de natureza bibliográfica, a acerca da prevalência dos transtornos mentais comuns e seus fatores de risco. O levantamento dos artigos foi realizado nas seguintes bases de dados: Scientific Electronic Library Online (Scielo) e Literatura Latino-americana e do Caribe em Ciências da Saúde (IILACS) de forma pareada durante os meses 


\section{artigo}

Santos, A.L.T.; Souza, J.F.; Ribeiro, C.L.; Nascimento, P.A.C.; Bruno, T.; Cecilia, H.

Prevalência de transtornos mentais menores em estudantes da área da saúde e os fatores relacionados

de Janeiro á Fevereiro de 2021. Para a busca dos dados utilizou-se os seguintes descritores indexados no DeCS (Descritores da Ciência em Saúde): "Saúde mental", "Universitário", "Distúrbios emocionais", "Fatores de risco" e "Transtorno mental". Foram realizadas pesquisas com múltiplas combinações entre os descritores em associação com o operador booleano And.

Para a seleção dos artigos foi utilizado os seguintes critérios de inclusão: artigos publicados no período de 2016 a 2021 , artigos disponíveis online nas bases de dados, textos completos e gratuitos, publicados em português, relacionada á temática. Dos critérios de exclusão: artigos duplicados em mais de uma base de dados e que não respondem á questão norteadora desse estudo, além de excluir dissertações, dossiês, carta ao autor, teses, estudos de revisão de literatura, Trabalho de Conclusão de Curso e aqueles que não descreviam rigorosamente o método.

Para a construção da Revisão Integrativa da Literatura foi seguido às quatro etapas propostas 7 , a saber, $1^{\text {a }}$ etapa da coleta de dados iniciou com a leitura do título e do resumo, na $2^{a}$ etapa foram lidas a metodologia e os resultados do artigo, na $3^{\text {a }}$ etapa foi feita uma leitura aprofundada dos resultados, destacando aqueles que respondiam ao objetivo proposto por este estudo, na $4^{a}$ etapa foi feita uma revisão dos artigos para a síntese de conhecimentos, onde foi concebido um resumo de resultado principal. A seguir, o fluxograma explicita os procedimentos de coleta dos dados (Figura 1).

\section{RESULTADOS}

A seleção dos artigos foi realizada mediante a leitura criteriosa do título, resumo
Figura 1: Fluxograma representando o processo de coletas de dados, Juazeiro do Norte, Ceará, Brasil.

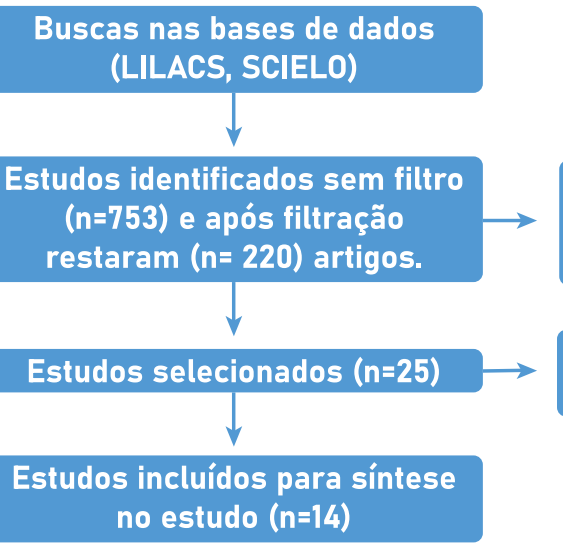

Fonte: Autoria própria com base em PEREIRA E GALVÃO (2014) e texto na íntegra, com o objetivo de responder a questão norteadora. Na sequência foram delimitadas as variáveis para análise, como: metodologia abordada, ano de publicação, cenários dos estudos, bem como o enfoque temático para análise e discussão dos dados. Dessa forma, foram definidas as seguintes categorias: Categoria I- Prevalência dos transtornos mentais comuns em universitário da área da saúde e Categoria II- Fatores associados ao risco de depressão e ansiedade no ambiente universitário.

Identificaram-se 753 publicações, 129 artigos na base de dados da scientific electronic library online (scielo), e 624 na base dados do literatura latino-americana e do caribe em ciências da saúde (lilacs). Após a aplicação dos critérios como ano de publicação e idioma, permaneceram 82 estudos na scientific electronic library online (scielo) e 138 no literatura latino-americana e do caribe em ciências da saúde (lilacs). Destes, foram excluídos 40 artigos duplicados, 65 não respondiam a temática do estudo, 45 foram excluídos através do titulo, 25 excluídos por serem de natureza bibliográfica e 20 por conta do resumo. Permaneceram 25 estudos, porém, após uma leitura minuciosa dos artigos excluiu-se 11. Assim, de 14 artigos compuseram amostra final.

\section{DISCUSSÃO}

\section{Categoria I - Prevalência dos trans- tornos mentais comuns em universi- tário da área da saúde.}

De acordo com o estudos realizados 8 , mostra a alta prevalência de sintomas ansiosos, depressivos e de estresse em estudantes universitários, inclusive são maiores

Quadro 1: Caracterização dos artigos encontrados de acordo com o autor, título, tipo de estudo, objetivos, resultados e bases de dados, Juazeiro do Norte, Ceará, Brasil, 2021.

\begin{tabular}{|l|c|c|c|c|}
\hline AUTOR/ANO & TÍTULO & TIPO DE ESTUDO & OBJETIVOS & RESULTADO \\
\hline Arruda, ES et & $\begin{array}{c}\text { Avaliação dos graus de ansie- } \\
\text { dade em acadêmicos de uma } \\
\text { al. } 2020\end{array}$ & $\begin{array}{c}\text { Pesquisa de cor- } \\
\text { te transversal com } \\
\text { abordagem descritiva, } \\
\text { quantitativa e observa- } \\
\text { cional. }\end{array}$ & $\begin{array}{c}\text { Avaliar o grau de ansie- } \\
\text { dade dos acadêmicos } \\
\text { de uma instituição de } \\
\text { ensino superior privada. }\end{array}$ & $\begin{array}{c}\text { amostra, } 62,3 \% \text { apresentou algum } \\
\text { grau de ansiedade, sendo os cur- } \\
\text { sos da área de saúde os que mais } \\
\text { apresentaram estudantes com } \\
\text { graus de ansiedade. }\end{array}$ \\
\hline
\end{tabular}




\begin{tabular}{|c|c|c|c|c|}
\hline $\begin{array}{l}\text { Guedes, AF } \\
\text { et al. } 2019\end{array}$ & $\begin{array}{l}\text { Prevalência e correlatos da } \\
\text { depressão com características } \\
\text { de saúde e demográficas de } \\
\text { universitários de medicina. }\end{array}$ & $\begin{array}{l}\text { Estudo descritivo, } \\
\text { transversal, } \\
\text { com abordagem } \\
\text { quantitativa. }\end{array}$ & $\begin{array}{c}\text { Avaliar a prevalência } \\
\text { de depressão e } \\
\text { correlacioná-la com } \\
\text { características de } \\
\text { saúde e demográficas } \\
\text { de estudantes do curso } \\
\text { de medicina de uma } \\
\text { faculdade do interior do } \\
\text { nordeste brasileiro. }\end{array}$ & $\begin{array}{c}\text { Foi observado que a prevalência de } \\
\text { depressão nos estudantes foi de } \\
52,8 \% \text {, distribuídos em depressão } \\
\text { leve }(39,1 \%) \text {, moderada }(12,3 \%) \text { e } \\
\text { grave }(1,4 \%) \text {. Maiores pontuações } \\
\text { de depressão em mulheres }(r= \\
0,24 ; p<0,01)\end{array}$ \\
\hline $\begin{array}{l}\text { Lima, SO et } \\
\text { al. } 2019\end{array}$ & $\begin{array}{l}\text { Prevalência da Depressão nos } \\
\text { Acadêmicos da Área de Saúde. }\end{array}$ & $\begin{array}{l}\text { Abordagem quali-quan- } \\
\text { titativo. }\end{array}$ & $\begin{array}{c}\text { O artigo analisa os } \\
\text { diferentes graus da } \\
\text { depressão nos cursos da } \\
\text { área de saúde e correla- } \\
\text { cionar esse transtorno } \\
\text { ao gênero e à idade. }\end{array}$ & $\begin{array}{l}\text { Nota-se pelo BDI que os sintomas } \\
\text { depressivos entre estudantes da } \\
\text { área da saúde têm-se mostrado } \\
\text { superior a outras populações de } \\
\text { idade correspondente }\end{array}$ \\
\hline $\begin{array}{l}\text { Ribeiro, IBS } \\
\text { et al. } 2020\end{array}$ & $\begin{array}{l}\text { Impacto da autoestima e dos } \\
\text { fatores sociodemográficos na } \\
\text { autoeficácia de estudantes de } \\
\text { graduação em enfermagem }\end{array}$ & $\begin{array}{l}\text { Pesquisa com tipo de } \\
\text { estudo transversal. }\end{array}$ & $\begin{array}{l}\text { Identificar os níveis de } \\
\text { autoeficácia e autoes- } \\
\text { tima em estudantes de } \\
\text { graduação em enferma- } \\
\text { gem e verificar a relação } \\
\text { destes constructos } \\
\text { entre si e com variáveis } \\
\text { sociodemográficas. }\end{array}$ & $\begin{array}{c}\text { A autoeficácia foi associada ao } \\
\text { sexo masculino, opção prioritária } \\
\text { no vestibular pela enfermagem, } \\
\text { satisfação com o curso e ausência } \\
\text { de sobrecarga, além de correlacio- } \\
\text { nar-se positivamente com a idade } \\
\text { e autoestima. }\end{array}$ \\
\hline $\begin{array}{l}\text { Flesch, BD et } \\
\text { al. } 2020\end{array}$ & $\begin{array}{c}\text { Episódio depressivo maior en- } \\
\text { tre estudantes universitários } \\
\text { do Sul do Brasil. }\end{array}$ & $\begin{array}{c}\text { Estudo censitário trans- } \\
\text { versal. }\end{array}$ & $\begin{array}{c}\text { Este estudo avalia a } \\
\text { prevalência e os fatores } \\
\text { associados à depressão } \\
\text { maior em universitários, } \\
\text { com ênfase na influência } \\
\text { do meio acadêmico, da } \\
\text { área de estudo escolhida } \\
\text { e do ambiente em que } \\
\text { estão inseridos. }\end{array}$ & $\begin{array}{c}\text { Observou-se que um total de } 32 \% \\
\text { dos universitários tem episódio } \\
\text { depressivo maior, o problema foi } \\
\text { mais frequente entre pertencente } \\
\text { ao sexo feminino, de } 21 \text { a } 23 \text { anos } \\
\text { de idade com histórico familiar } \\
\text { de depressão que moravam com } \\
\text { amigos. }\end{array}$ \\
\hline $\begin{array}{l}\text { Lelis, KCG et } \\
\text { al. } 2020\end{array}$ & $\begin{array}{l}\text { Sintomas de depressão, an- } \\
\text { siedade e uso de medicamen- } \\
\text { tos em universitários. }\end{array}$ & $\begin{array}{l}\text { Estudo descritivo, } \\
\text { transversal, de análise } \\
\text { quantitativa. }\end{array}$ & $\begin{array}{l}\text { Avaliar os sintomas de } \\
\text { ansiedade e depressão e } \\
\text { o uso de medicamentos } \\
\text { em universitários. }\end{array}$ & $\begin{array}{l}\text { Foi observado que os sintomas } \\
\text { de depressão e ansiedade foram } \\
\text { identificados em } 52,3 \% \text { ( } n=153 \text { ) e } \\
41,1 \% \text { ( } n=120 \text { ) dos participantes, } \\
\text { respectivamente. }\end{array}$ \\
\hline $\begin{array}{l}\text { Leão, AM et } \\
\text { al. } 2018\end{array}$ & $\begin{array}{l}\text { Prevalência e Fatores Asso- } \\
\text { ciados à Depressão e Ansie- } \\
\text { dade entre Estudantes Uni- } \\
\text { versitários da Área da Saúde } \\
\text { de um Grande Centro Urbano } \\
\text { do Nordeste do Brasil. }\end{array}$ & $\begin{array}{l}\text { Estudo transversal } \\
\text { analítico. }\end{array}$ & $\begin{array}{l}\text { Estimar a prevalência e } \\
\text { os fatores associados à } \\
\text { depressão e ansiedade } \\
\text { em estudantes universi- } \\
\text { tários da área da saúde. }\end{array}$ & $\begin{array}{l}\text { Observou-se que as prevalências } \\
\text { de depressão e ansiedade foram } \\
\text { de } 28,6 \% \text { e } 36,1 \% \text {, respectivamen- } \\
\text { te. Estudantes menos satisfeitos } \\
\text { com o curso apresentaram chance } \\
\text { quase quatro vezes maior de } \\
\text { terem depressão. }\end{array}$ \\
\hline $\begin{array}{l}\text { Fernandes, } \\
\text { MA et al. } \\
2018\end{array}$ & $\begin{array}{l}\text { Prevalência de sintomas an- } \\
\text { siosos e depressivos em estu- } \\
\text { dantes universitários de uma } \\
\text { instituição pública. }\end{array}$ & $\begin{array}{l}\text { Estudo censitário, } \\
\text { transversal e analítico. }\end{array}$ & $\begin{array}{l}\text { Identificar a prevalência } \\
\text { de sintomas ansiosos e } \\
\text { depressivos e suas cor- } \\
\text { relações com caracterís- } \\
\text { ticas sociodemográficas. }\end{array}$ & $\begin{array}{l}\text { Observa-se que a prevalência mé- } \\
\text { dia de depressão em universitários } \\
\text { é de 30,6\%, enquanto na popula- } \\
\text { ção não universitária é de } 9 \% \text {. }\end{array}$ \\
\hline $\begin{array}{l}\text { Andrade, AS } \\
\text { et al. } 2016\end{array}$ & $\begin{array}{l}\text { Vivências Acadêmicas e Sofri- } \\
\text { mento Psíquico de Estudantes } \\
\text { de Psicologia. }\end{array}$ & $\begin{array}{c}\text { Pesquisa transversal de } \\
\text { abordagem qualitativa e } \\
\text { quantitativa. }\end{array}$ & $\begin{array}{l}\text { Este artigo apresenta } \\
\text { dados de uma pes- } \\
\text { quisa cujo objetivo era } \\
\text { o levantamento das } \\
\text { vivências acadêmicas } \\
\text { dos estudantes. }\end{array}$ & $\begin{array}{l}\text { Nas questões abertas referen- } \\
\text { tes às vivências e ao sofrimento } \\
\text { psíquico foram apresentados } \\
\text { indicadores explíitos de mal-estar } \\
\text { universitário. }\end{array}$ \\
\hline
\end{tabular}




\begin{tabular}{|c|c|c|c|c|}
\hline $\begin{array}{l}\text { Ribeiro, IBS } \\
\text { et al. } 2020\end{array}$ & $\begin{array}{c}\text { Prevalência de Fatores Asso- } \\
\text { ciados à Depressão e Ansie- } \\
\text { dade em Estudantes de Medi- } \\
\text { cina Brasileiros }\end{array}$ & $\begin{array}{c}\text { Estudo do tipo trans- } \\
\text { versal. }\end{array}$ & $\begin{array}{l}\text { 1. estimar a prevalência } \\
\text { de escores indicativos de } \\
\text { ansiedade, depressão e } \\
\text { ansiedade e } 2 \text {. analisar } \\
\text { os fatores associados } \\
\text { com tais condições em } \\
\text { estudantes. }\end{array}$ & $\begin{array}{l}\text { Foi observado nesse estudo } \\
\text { que a ansiedade foi a condição } \\
\text { mais comum }(41,4 \%) \text {, seguida de } \\
\text { depressão }(8,2 \%) \text { e de depressão e } \\
\text { ansiedade simultâneas }(7,0 \%) \text {. }\end{array}$ \\
\hline $\begin{array}{l}\text { Cardoso, JV } \\
\text { et al. } 2019\end{array}$ & $\begin{array}{l}\text { Estresse em estudantes uni- } \\
\text { versitários: uma abordagem } \\
\text { epidemiológica. }\end{array}$ & $\begin{array}{l}\text { Trata-se de estudo } \\
\text { quantitativo, descritivo, } \\
\text { epidemiológico trans- } \\
\text { versal. }\end{array}$ & $\begin{array}{l}\text { Identificar a ocorrência } \\
\text { de estresse e as vulne- } \\
\text { rabilidades sociodemo- } \\
\text { gráficas e acadêmicas } \\
\text { em estudantes univer- } \\
\text { sitários. }\end{array}$ & $\begin{array}{l}\text { Esse estudo revelou que a ocor- } \\
\text { rência de estresse em todos os } \\
\text { participantes deste estudo, com } \\
\text { média de estresse de } 26,18 \text { e } \\
\text { moda } 28 \text {, sendo que } 192(49,1 \%) \\
\text { estudantes universitários apre- } \\
\text { sentaram escore para nível de } \\
\text { estresse acima da média de todos } \\
\text { os participantes. }\end{array}$ \\
\hline $\begin{array}{l}\text { Ariño, DO; } \\
\text { Bardagi, MP, } \\
2018\end{array}$ & $\begin{array}{c}\text { Relação entre Fatores Aca- } \\
\text { dêmicos e a Saúde Mental de } \\
\text { Estudantes Universitários. }\end{array}$ & $\begin{array}{l}\text { Trata-se de uma pes- } \\
\text { quisa correlacional, de } \\
\text { caráter descritivo, com } \\
\text { corte transversal. }\end{array}$ & $\begin{array}{c}\text { Analisar as relações } \\
\text { entre ansiedade, de- } \\
\text { pressão e stress com a } \\
\text { qualidade das vivências } \\
\text { acadêmicas e a autoe- } \\
\text { ficácia. }\end{array}$ & $\begin{array}{l}\text { Observou-se que as Vivências } \\
\text { Acadêmicas apresentaram rela- } \\
\text { ções negativas, estatisticamente } \\
\text { significativas com as dimensões } \\
\text { da Vulnerabilidade Psicológica. }\end{array}$ \\
\hline $\begin{array}{l}\text { Mesquita, } \\
\text { AM et al. } \\
2016\end{array}$ & $\begin{array}{c}\text { Depressão entre estudantes } \\
\text { de cursos da área da saúde de } \\
\text { uma universidade em mato } \\
\text { grosso }\end{array}$ & $\begin{array}{l}\text { Trata-se de um estudo } \\
\text { transversal, descritivo } \\
\text { com abordagem quan- } \\
\text { titativa. }\end{array}$ & $\begin{array}{l}\text { Identificar a tendência } \\
\text { depressiva entre aca- } \\
\text { dêmicos dos cursos de } \\
\text { saúde de uma universi- } \\
\text { dade pública. }\end{array}$ & $\begin{array}{l}\text { Observou-se que a tendência á } \\
\text { depressão esteve presente em } \\
\text { 41\% dos universitários, sendo mais } \\
\text { relevante no curso de enferma- } \\
\text { gem (55\%). }\end{array}$ \\
\hline $\begin{array}{l}\text { Lameu, JN; } \\
\text { Salazar, TL; } \\
\text { Souza, WF, } \\
2016 .\end{array}$ & $\begin{array}{l}\text { Prevalência de sintomas de } \\
\text { stress entre graduandos de } \\
\text { uma universidade pública. }\end{array}$ & $\begin{array}{c}\text { Trata-se de um estudo } \\
\text { transversal. }\end{array}$ & $\begin{array}{c}\text { Avaliar a prevalência } \\
\text { de sintomas de stress } \\
\text { entre os estudantes de } \\
\text { graduação. }\end{array}$ & $\begin{array}{l}\text { Foi observado que a prevalência } \\
\text { de stress encontrada foi de } 50 \% \text {, } \\
\text { estando a maior parte desses } \\
\text { alunos na fase de resistência. }\end{array}$ \\
\hline
\end{tabular}

que as encontradas na população em geral. A morbidez psicológica não causa prejuízo apenas no contexto da saúde, desempenho e qualidade de vida dos universitários, mas também pode levar complicações importantes e significativas no ambiente familiar. Visto isso, análises nacionais e internacionais manuseando distintos instrumentos voltados á investigação da saúde mental, tem apontado a vulnerabilidade da população universitária à ocorrência de sinais e sintomas psicopatológicos.

Estudos apontam que universitários da área da saúde estão mais predispostos a desenvolver TMC (transtornos mentais comuns) em comparação a universitários de outros cursos. O aparecimento de transtornos mentais comuns no ambiente acadêmico pode ser observado logo após o aluno ingressar na universidade, sendo mais recorrente em universitários da área da saúde, pois estes tem que aprender diariamente a lidar com a dor'. Afirma que a depressão é mais prevalente entre os cursos de medicinas e outras áreas da saúde ${ }^{10}$.

Diante dos estudos, foi visto que existe uma divergência entre alguns autores diante da prevalência do TMC entre universitários de cursos da área da saúde. Os níveis mais altos de ansiedade e depressão estão entre os estudantes do curso medicina do sexo feminino ${ }^{11}$. Os principais distúrbios psíquicos desencadeados em um ambiente universitários é a depressão e a ansiedade. Sendo que entre estudantes de medicina a maior evidencia foi á ansiedade com $(41,4 \%)$, seguida de depressão $(8,2 \%)$ e de depressão e ansiedade simultâneas (7,0\%). Ainda com mesmo autor, a prevalência de depressão entre os estudantes ao todo foi de $28,6 \%$, variando variados entre sintomas leve, moderados e graves. Já a preva- 
lência da ansiedade entre os estudantes foi de $36,1 \%{ }^{12}$.

$\mathrm{O}$ índice de TMC principalmente depressão e ansiedade encontrados nos estudantes universitários são altos, inclusive superiores ao encontrados na população em geral, sendo que os alunos dos primeiros anos de faculdade apresentam maior grau sintomas ansiosos e depressivos. Foi feita uma analise de dados a partir de questionários e foi observado que a depressão era o distúrbio mental com maior prevalência. Desses alunos 14\% sofriam de sintomas de depressão, $3 \%$ com sintomas ansiosos e 3\% distúrbios alimentares. Ainda com o mesmo autor, pode-se evidenciar que a depressão ela prejudica o alunos, pois influencia significativamente no baixo desempenho acadêmico ${ }^{13}$.

\section{Categoria II - Fatores associados ao} risco de depressão e ansiedade no ambiente universitário.

$\mathrm{O}$ ingresso na universidade pode submeter os estudantes a estressores específicos deixando essa população vulnerável ao desenvolvimento de alguns transtornos mentais como, ansiedade, depressão e o estresse. A saúde mental seria uma estabilidade mental entre sentimentos internos e experiências externas. $\mathrm{O}$ excesso de trabalhos demanda acadêmica e incertezas do futuro profissional podem gerar um estado de fadiga física e psíquica, devastando o equilíbrio mental e tornando o indivíduo aflito, deprimido, nervoso e neurótico ${ }^{14}$.

Os transtornos mentais comuns que envolvem a população universitária são adquiridos muitas vezes através de pressão acadêmica e sobrecarga, tendo como elementos principais, a fadiga, insônia, a irritabilidade e a dificuldade na concentração 15. Estudos apontam que $18,5 \%$ a $44,9 \%$ dos estudantes universitários brasileiros, principalmente da área da saúde, apresentam variações de $\mathrm{TMC}^{2}$.

Os fatores como insatisfação com curso, quantidade insuficiente de horas de sono, relacionamento insatisfatório com colegas do curso e com docentes estão associados a maior prevalência de depressão entre os estudantes. Destacou também
O ingresso na

universidade

pode submeter

os estudantes

a estressores

específicos

deixando essa

população

vulnerável ao

desenvolvimento

de alguns

transtornos mentais

como, ansiedade,

depressão e o

estresse. A saúde

mental seria uma

estabilidade mental

entre sentimentos

internos $\mathrm{e}$

experiências

externas. que religião, renda, relação com colegas de residência, faixa etária, forma de pagamento do curso, se bebe, se fuma, estado civil são fatores que não apresentam associações significativas com a presença de depressão. Já no caso da ansiedade fatores como o sexo feminino, insônia, preocupação com o futuro, não praticar atividade física, relacionamento insatisfatório com familiares, amigos e colegas estão associado significativamente com a prevalência do quadro de ansiedade ${ }^{16}$.

Estudantes da área da saúde apresentam maior prevalência de adoecimento mental, e mencionou que um dos fatores de risco para TMC dessa população é o período do curso no qual o aluno se encontra, tendo em vista que o período inicial é marcado pela substituição do ensino médio para o superior e pelo primeiro contato com a prática profissional, enquanto o último período é marcado pelo processo de abandono do papel de estudante e a entrada no mercado de trabalho. As demandas acadêmicas também são fatores significativos que podem causar impactos a saúde mental desse grupo, aspectos como a elevada carga horária de estudo, ${ }^{17}$ recursos financeiros, gastos com trabalhos, falta de tempo para lazer, qualidade do sono, e carga horária do curso o grau de exigência no processo de formação, e novas demandas de planejamento de tempo e de estudo, tornam-se pontos de estresse que são vivenciados com grande intensidade pelo aluno. ${ }^{18}$

\section{CONCLUSÃO}

Esta revisão possibilitou evidenciar a prevalência dos TMC entre os estudantes universitários, mostrando que a depressão e a ansiedade estão em alta prevalência. A análise dos fatores de risco que mais se relacionou ao sofrimento psíquico entre os universitários foi á pressão acadêmica e sobrecarga, insatisfação com o curso, relacionamentos insatisfatórios com os colegas e docentes, residir longe da família, além da vida acadêmica e relacionais sendo elas as que mais frequentemente associaram-se a presença de sofrimento psíquica entre os 
universitários. É importante a realização de mais estudos dessa natureza tendo em vista a prevalência de fatores depressivos em universitários tendo caracterizado problemática que afeta todas as classes sociais e que requer de uma atenção maior.

\section{REFERÊNCIAS}

1. Carleto CT, Dias de Moura RC, Snatos VS, Kauchakje Pedrosa LA. Adaptação à universidade e transtornos mentais comuns em graduandos de enfermagem. Adaptação à universidade e transtornos mentais comuns em graduandos de enfermagem Revista eletrônica de enfermagem[Internet]. 2018 Apr 17 [cited 2021 Jan 26];20:1-11. Available from: https://revistas.ufg.br/fen/article/ view/43888.

2. Graner KM, Ramos cerqueira ATA. Revisão integrativa: sofrimento psíquico em estudantes universitários e fatores associados. Ciência \& Saúde Coletiva [Internet]. 2019 [cited 2021 Jan 26];24(4):1-20. DOI 10.1590/1413-81232018244.09692017. Available from: https:/www.scielo.br/scielo.php?script=sci_abst ract\&pid=S1413-81232019000401327\&lng=en\&nrm=iso\&tl$\mathrm{ng}=\mathrm{pt}$.

3. Cruz FL. Por que depressão e ansiedade afetam cada vez mais universitários [Internet]. [place unknown]: Desafios da Educação; 2018 Jul 30 [cited 2021 Jan 26]. Available from: https://desafiosdaeducacao.grupoa.com.br/ansiedade-e-depressao-na-universidade/.

4. Lopes CS. Como está a saúde mental dos brasileiros? A importância das coortes de nascimento para melhor compreensão do problema. Caderno de Saúde Pública [Internet]. 2020 [cited 2021 Jan 26];36(2):1-4. DOI 10.1590/0102-311X00005020. Available from: https://www.sanarmed.com/artigos-cientificos/como-esta-a-saude-mental-dos-brasileiros-a-importancia-das-coortes-de-nascimento-para-melhor-compreensao-do-problema

5. Fernandes MA, Vieira FER, Silva JS, Avelino FVSD, Santos JDM. Prevalência de sintomas ansiosos e depressivos em universitários de uma instituição pública. Rev Bras Enferm [Internet]. 2018;71(Suppl 5):2169-75. [Thematic Issue: Mental health] DOI: http://dx.doi.org/10.1590/0034-7167-2017-0752

6. First MB. Tratamento das doenças mentais [Internet]. [place unknown]: Manual MSD; 2017 [cited 2021 Jan 26]. Available from: https://www.msdmanuals.com/pt-pt/ casa/dist\%C3\%BArbios-de-sa\%C3\%BAde-mental/considera $\% C 3 \% A 7 \% C 3 \% B 5$ es-gerais-sobre-cuidados-com-a-sa\%C3\%BAde-mental/tratamento-das-doen\%C3\%A7as-mentais

7. Pereira, MG; Galvão, TF. Etapas de busca e seleção de artigos em revisões sistemáticas da literatura. Epidemiologia e Serviços de Saúde, [S.L.], v. 23, n. 2, p. 369-371, jun. 2014. FapUNIFESP (SciELO). http://dx.doi.org/10.5123/s1679-49742014000200019.

8. Padovani RC, Neufeld CB, Maltoni J, Barbosa LNF, Souza WF, Cavalcanti HAF, Lameu JN. Vulnerabilidade e bem-estar psicológicos do estudante universitário. Revista Brasileira de Terapias Cognitivas [Internet]. 2014 [cited 2021 Feb 5];10(1) DOI http://dx.doi.org/10.5935/1808-5687.20140002. Available from: http://pepsic.bvsalud.org/scielo.php?script=sci_arttext\&pid=S1808-56872014000100002\&lng=pt\&nrm=iso\&tlng=pt

9. Silva RS, Costa LA. Prevalência de transtornos mentais comuns entre estudantes universitários da área da saúde. Revista da psicologia [Internet]. 2015 Jul 15 [cited 2021 Feb 5];15(23) Available from: https://revista.pgsskroton.com/index.php/renc/article/ view/2473

10. Araújo CS. Avaliação da prevalência de sintomas característicos de ansiedade e depressão em estudantes da área da saúde. 2019. Available from: http://dspace.sti.ufcg.edu.br:8080/jspui/bitstream/riufcg/11914/1/CAMILA\%20SOARES\%20DE\%2OARAUJO\%20-\%20\%20TCC\%2OFARM\%C3\%81CIA\%20\%202019.pdf. Acesso em: 12 out. 2020.

11. Roberto A, Almeida A. saúde mental de estudantes de MEDICINA Estudo Exploratório na Universidade da Beira Interior.2011. Available from: file://C:/Users/Positivo/Downloads/1490-21401-PB.

12. Ribeiro IBS, CORREA MM, OLIVEIRA G, CADE NV. Transtorno mental comum e condição socioeconômica em adolescentes do Erica. Revista de Saúde Pública, 2020 jan 21, [s.l.], v. 54, p. 1-5, Universidade de Sao Paulo, Agencia USP de Gestao da Informacao Academica (AGUIA). http://dx.doi.org/10.11606/s15188787.2020054001197

13. Costa, Ma, Moreira,YB. Saúde mental no contexto universitário. Anais do Seminários Sobre Ensino em Design. 2016 out [S.L.], v. 2, n. 10, p. 1-7. Editora Edgard Blücher. http://dx.doi. org/10.5151/despro-sed2016-009.

14. Silva, AO, Cavalcante NJL. Associação entre níveis de atividade física e transtorno mental comum em estudantes universitários. Motricidade, 2014 mar 1, v. 10, n. 1, p. 1-11. Desafio Singular, Lda. http://dx.doi.org/10.6063/motricidade.10(1).2125.

15. Gomes CFM, Pereira J, Ronaldo J, Cardoso JV, Silva DA. Transtornos mentais comuns em estudantes universitários. Smad Revista Eletrônica Saúde Mental Álcool e Drogas (edição em Português), 2020 fev, v. 16, n. 1, p. 1-8, 21 fevUniversidade de Sao Paulo, Agencia USP de Gestao da Informacao Academica (AGUIA). http://dx.doi.org/10.11606//issn.1806-6976.smad.2020.157317.

16. Leão AM, Gomes IP, Ferreira MJM, Cavalcanti LPG. Prevalência e Fatores Associados à Depressão e Ansiedade entre Estudantes Universitários da Área da Saúde de um Grande Centro Urbano do Nordeste do Brasil. Revista Brasileira de Educação Médica, 2018 dez [s.l.], v. 42, n. 4, p. 55-65. FapUNIFESP (SciELO). http://dx.doi. org/10.1590/1981-52712015v42n4rb20180092.

17. Seltenreich , LS; Galante, MS; Barea, CAA; Silva, LP; Morais JSL A. Qualidade de vida dos graduandos de enfermagem: Uma revisão integrativa. Saúde Coletiva (Barueri),9(50), 1837- 1841. Recuperado de htt://revistas.mpmcomunicacao.com.br/index. php/ saudecoletiva/article/view/166.

18. Ariño OD, Bardagi MP. Relação entre Fatores Acadêmicos e a Saúde Mental de Estudantes Universitários. Revista Psicologia em Pesquisa, $2018 \mathrm{dez} 28$, [s.l.], v. 12,p. 1-3. Universidade Federal de Juiz de Fora. http://dx.doi.org/10.24879/2018001200300544 